\title{
Wireless charging scheme for medium power range application systems
}

\author{
D Karthikeyan, Sayon Koley, Mayukh Bagchi, Avijit Bhattacharya, K Vijayakumar \\ Department of Electrical and Electronics Engineering, SRM Institute of Science and Technology, India
}

\begin{tabular}{l} 
Article Info \\
\hline Article history: \\
Received Apr 13, 2020 \\
Revised Jun 10, 2020 \\
Accepted Jul 2, 2020 \\
\hline Keywords: \\
Digital signal processor (DSP) \\
Full bridge class D Inverter \\
Inductive coupling \\
Inductive power transfer (IPT) \\
Integrated circuit (IC) \\
Lithium polymer (LiPo) \\
Near field transmission \\
Receiver coil (Rx) \\
Resonant inverter \\
Transmission coil (Tx) \\
Wireless power transfer (WPT)
\end{tabular}

\begin{abstract}
Wireless power transmission (WPT) has attracted a wide variety of subjects in various disciplines and has also become a highly active research field due to its capacity to facilitate charging systems. Wireless power transmission will be compulsory to use soon as this technology enables electrical energy to be transmitted from a power source to an electrical load over an air gap without connecting wires. Wireless power transmission has been developed in the low power ( $1 \mathrm{~W}$ to $10 \mathrm{~W})$ and high power $(100 \mathrm{~W}-500 \mathrm{~W})$ region. While the low power region development focuses on powering medical transplants and mobile charging, the higher end of the power spectrum is being developed for the electric vehicle (EV) applications. However medium power range (10W to $100 \mathrm{~W}$ ) is relatively unexplored due to lack of proper applications. The commercial WPT scheme is mainly used for the charging of lithium-ion batteries. Sensitive medium power loads like Lithium Polymer (LiPo) batteries do not have a wireless modular charging system. This paper discusses a proposed scheme for wireless charging of medium-range loads. LiPo batteries are used as the targeted charging load. A minimalistic approach has been considered while designing the electronics for efficiency improvement and a compact, modular scheme. The proposed scheme has been developed for drone and robotics applications and the results are validated.
\end{abstract}

This is an open access article under the CC BY-SA license.

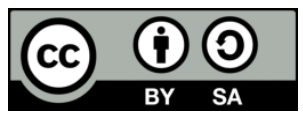

\section{Corresponding Author:}

D Karthikeyan,

Department of Electrical and Electronics Engineering,

SRM Institute of Science \& Technology, Kattankulathur, Tamil Nadu, India.

Email: karthipncl@gmail.com

\section{INTRODUCTION}

Wireless technology makes it easy for users to travel wide distances without any hassles. Another physical disadvantage of wired technology is that cables can easily be broken, which is not an issue with wireless technology [1]. Wireless Power Transfer (WPT) allows power to be transmitted through an air gap without the need for load-bearing cables. WPT may provide compatible batteries or computers with power from an AC source, without physical connectors or wires. WPT will recharge cell phones and laptops, aircraft, bikes, and even transportation equipment. Wireless transmission of the power collected by solarpanel arrays in space may even be possible [2,3]. WPT has been an exciting development to replace wired chargers in consumer electronics. Nevertheless, since the late 1890s, there has been around the idea of power transmission without wires. Nikola Tesla was able to wirelessly light electrical bulbs using electrodynamic induction (aka resonant inductive coupling) in his Colorado Springs Institute [4]. The world is moving towards automation. WPT will be an important part of automating electrical and electronic items used in current times. Charging chords require human intervention which prevents full automation. Hence to automate drone services, vacuum cleaner, robotic equipment a WPT charging module is a requirement [5]. 
LiPo batteries are sensitive devices that require balanced charging methods. The current WPT technology is aimed at charging LioN batteries [6-7]. The chemical electrolyte between their positive and negative electrodes is the most important difference between lithium-ion and lithium-polymer batteries.

\section{WIRELESS PHYSICS}

Many electromagnetic effects can wirelessly transfer energy from one point to the next. The optimum approach is determined by the ideal distance between the source and device relative to two longitudinal scales: (1) the source or device dimension, and (2) the wavelength corresponding to the oscillation frequency [8]. Short-range transmission occurs over distances much smaller than the size of the source or device, a mid-range transfer occurs over distances greater than the source or device and long-range transfer occurs over distances far larger than the wavelength [9].

Figure 1 shows a wireless power transfer system with two magnetically coupled coils. The electric energy in the transmitter coil is transformed into magnetic energy that is stored in the receiver coil where it is converted back to electrical energy [10].

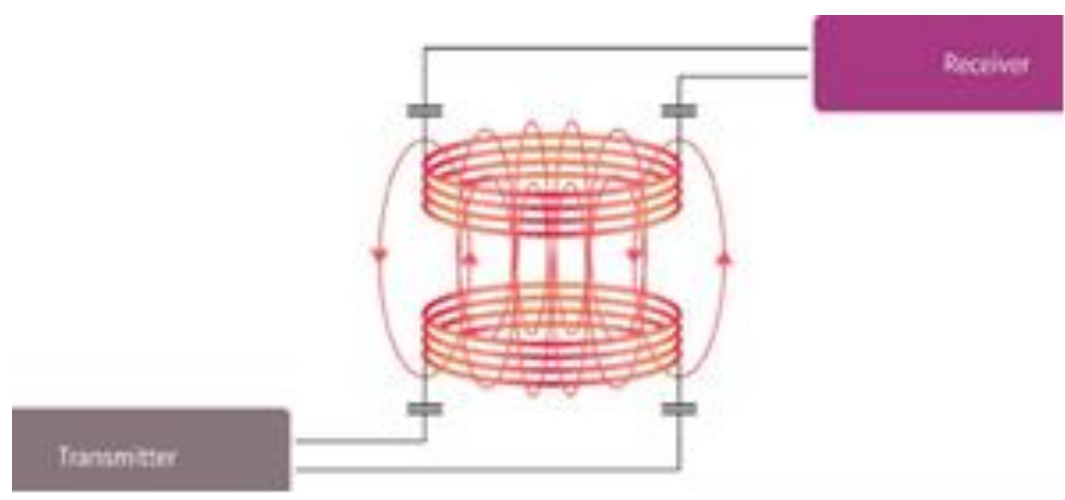

Figure 1. Resonant wireless power transfer concept

Wireless inductive power transfer (WPT) is limited to only a few millimeters of the distance between the transmitter and the receiver. With gaps in the range of centimeters, coupling decreases, and with it the efficiency of energy transfer decreases [11-12].

\subsection{System scheme}

The existing model used for wireless power transmission usually is comprised of the transmitter side circuit, receiver side circuit, a BMS, and a LioN battery as shown below in Figure 2. The existing model has two major drawbacks i.e sufficient power cannot be transferred due to the absence of a proper amplifying component like a MOSFET and also the output contains a lot of unwanted noises which affects the overall efficiency of the scheme. The proposed system can be separated into 3 components or subsystems- the inverter, the transmitter/ receiver, and finally the battery management system (BMS). These systems are interconnected as shown in Figure 3. The components, topologies have been selected for handling medium power range load requirements. The source of the inverter stage is direct current (D.C.) for this scheme. It can be taken from a $12 \mathrm{~V}, 2 \mathrm{~A}$ rated switched-mode power supply adapter.

The D.C source provides input to a full- bridge Class D inverter. It consists of 4 power switches, two in each leg. As the frequency of switching is high, MOSFET (Metal Oxide Field Effect Transistor) switches have been used for better switching response $[13,14]$. The output of the H- Bridge is provided to an LC resonant tank circuit, the inductive coil is the transmitter itself. The capacitor value to be set depends on the resonant frequency of power transfer [15]. The resonant frequency is the same as the switching frequency in this circuit [16]. The receiver coil will have a capacitor connected as per the decided frequency of power transfer. The next system is the BMS which involves the rectifier stage and necessary protection circuitrythe output of which is finally provided to the LiPo battery. A balanced charger is integrated into the BMS stage for the safe charging of the battery $[17,18]$. 


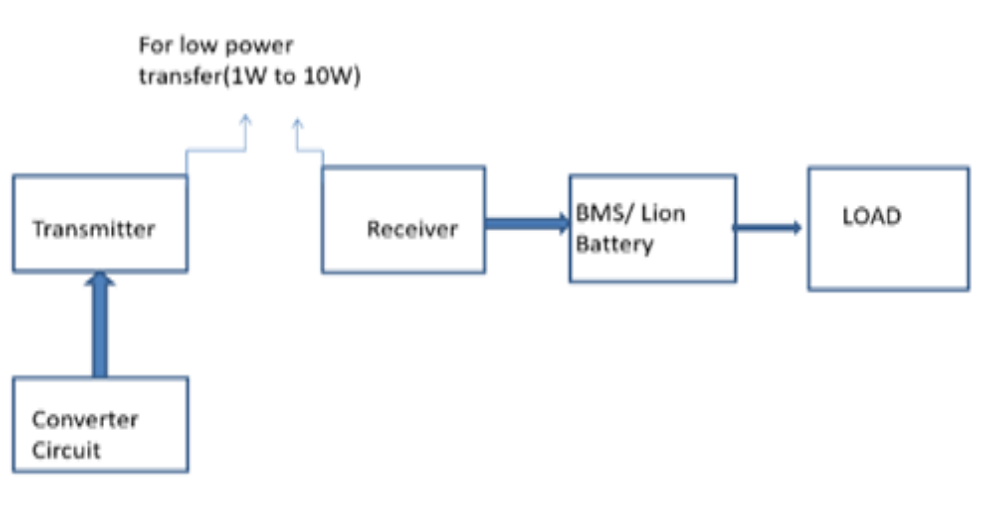

Figure 2. Block diagram of the existing scheme

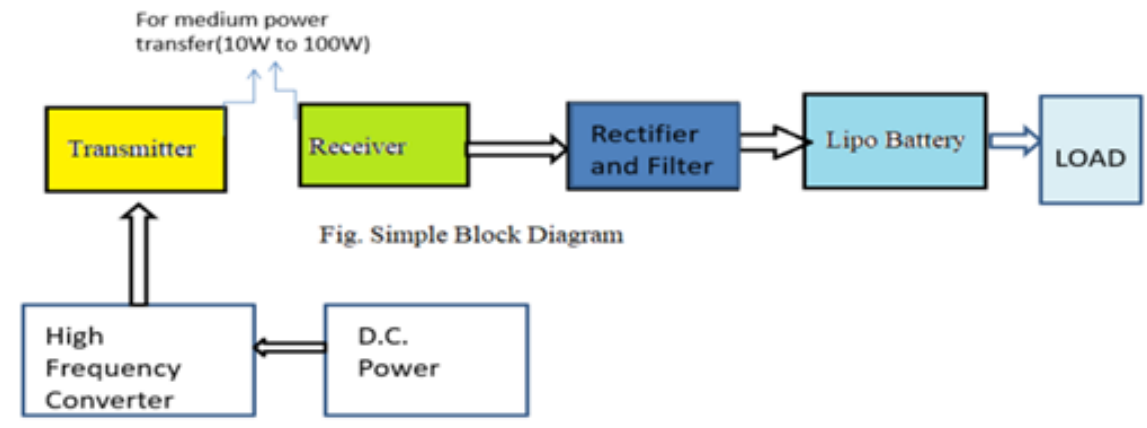

Figure 3. Block diagram of the proposed scheme

\subsection{Inverter switching scheme}

The topology used here is a basic H- Bridge inverter. The power switches have been controlled using a timer circuit. In a pulse wave, the duty cycle is defined as follows:

$$
\mathrm{D}=[\mathrm{TON} /(\mathrm{TON}+\mathrm{TOFF})]
$$

Where, $\mathrm{D}=$ duty cycle, $\mathrm{TON}=$ on period of Pulse, TOFF=OFF period of the pulse generated. In the simulation circuit, we are using a timer circuit that provides a 50\% duty cycle pulse wave to the driver IC of diagonal power switches of the full bridge. An inverted pulse wave is generated to drive the other two switches. A 555 timer circuit has been used for the implementation of the switching scheme and switching frequency of $150 \mathrm{kHz}$ is achieved. This switching scheme used is also called block control, where alternate switches are blocked in a leg to avoid shoot-through. The output obtained is a square wave having a voltage peak equal to the power supply voltage.

\subsection{Gate driving scheme}

TLP250 is a standalone IGBT / Mosfet driver IC. The input side consists of a GaAlA light-emitting diode. The output side gets a drive signal from an integrated photodetector. Consequently, the main feature is an electric separation between low and high power circuits. The electrical signals are transmitted optically through light. Users may use it to operate high voltage switch gate terminals in both configurations, such as high side operate and low side drive. It is available as a DIP kit with 8 pins.

\subsection{Simulation}

The simulation has been done using Infineon Designer-TINACloud is shown in Figure 4. It is an online prototyping platform incorporating the functionalities of analog and digital simulation in an Internet application. All the components used in the hardware are made by Infineon, so the use of this program for output analysis gave the ICs in the circuit an accurate behavior. The probing method used to measure the output across the dummy load is differential probing. The two-channel feed was connected across the load 
end and the ground was made common. The waveform received is shown in Figure 5. The black waveform is of the probe at the positive side of the load while the green waveform is of the second probe. The difference waveform is fed to the transmitter coil. The initial distortion is due to capacitance in the circuit. A setting of $15 \%$ has been used for rheostat to obtain the desired frequency of $150 \mathrm{kHz}$ for the simulation.

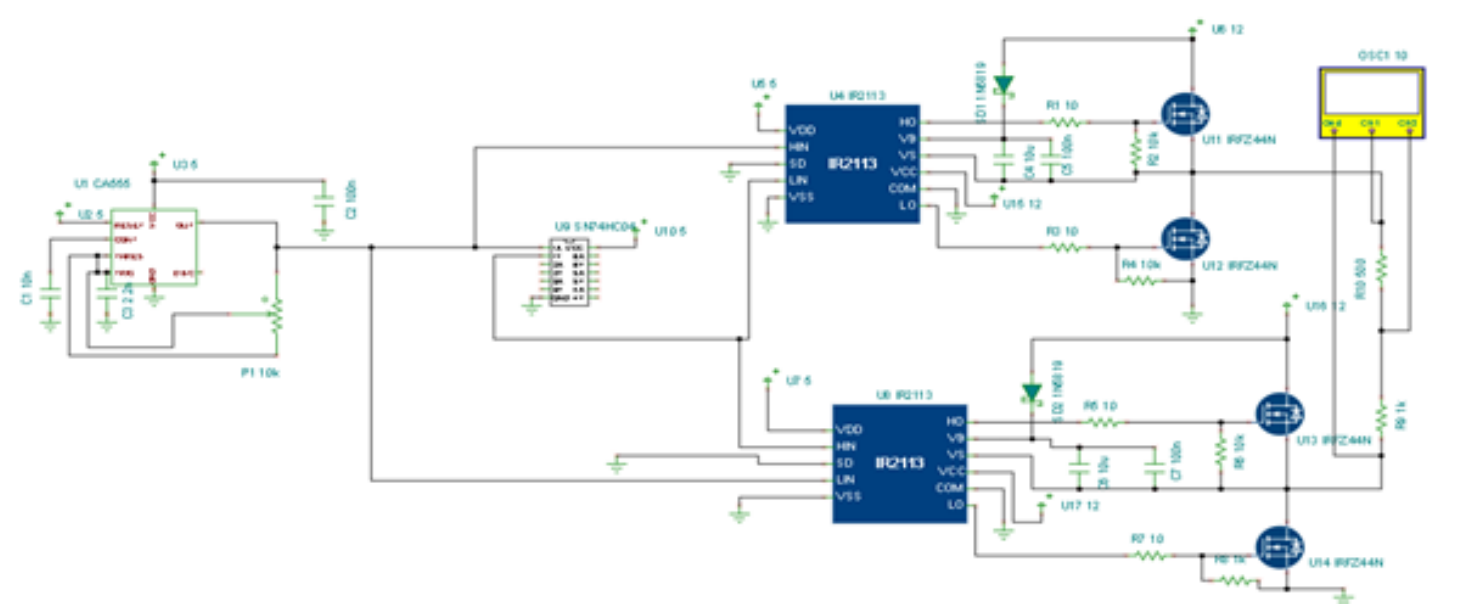

Figure 4. The simulated circuit scheme

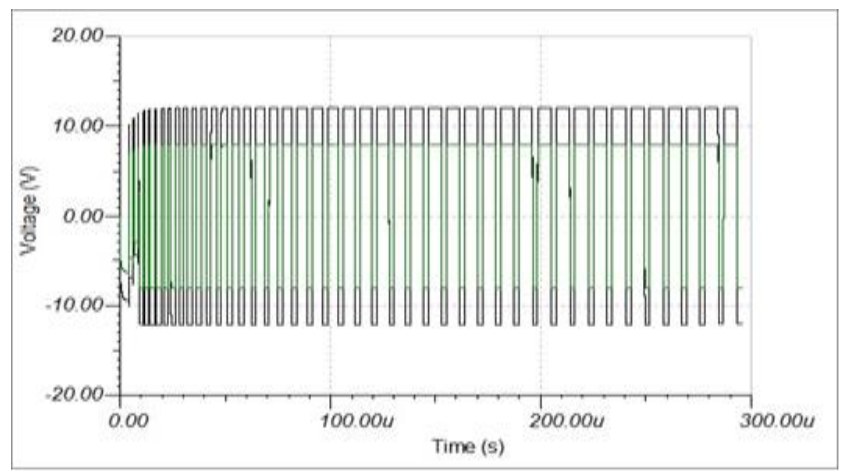

Figure 5. Voltage (vs.) time waveform at the transmitter side

\subsection{Component selection}

\subsubsection{Switching pulse generation}

A 28 pin DSP IC package dsPIC30F2010 is being used to provide the necessary pulses to the HBridge. It is a high-performance Microchip Digital Signal Transmitter. It has updated Harvard architecture and $\mathrm{C}$ compiler optimized instruction set architecture using 24-bit wide instructions, 16-bit wide data path, 12 Kbytes of on-chip Flash program space. This also has 512 bytes of RAM on-chip storage. It can support DC to $40 \mathrm{MHz}$ external clock input, and $4 \mathrm{MHz}-10 \mathrm{MHz}$ oscillator input with PLL specifications, making it suitable for this scheme. The load current requirement on the transmitter side has been set to $1.5-2 \mathrm{~A}$ and a voltage of $12 \mathrm{~V}$ is being used. Accordingly, the MOSFET switch specification criteria were set.

\subsubsection{Transmitter and receiver coils}

The transmitter coil is expected to handle a current of 1.5 to $2 \mathrm{~A}$ according to the load requirements and system design. Receiver coil has also been selected keeping the same range values, though it is expected to carry less current than transmitter due to the losses. The dimension of the square coil is shown in Figure 6 square coils are used as they are more efficient than circular ones [19]. High switching frequency produces higher-order harmonics and acoustic noises along with switching loss at edges [20, 21]. Hence a mediumrange operating frequency is chosen. Both the coils are rated at $20 \mathrm{~W}$ power handling capacity. The current rating of a device is limited by the heat produced in the dies and the resistance of the leads [22]. 
Based on discrete parts, electronic architecture offers advantages such as robust construction, versatility, and lower overall cost [23]. The larger the coil, the higher the effective distance induced by more magnetic fields between the transmitter coil and the receiver coil [24]. Yet bigger coils are bulky for high power range and not suitable for low power applications. If the inductance for a greater transfer distance is increased, the efficiency will decrease due to a higher loss of parasite resistance and an increase in the number of windings. [25].

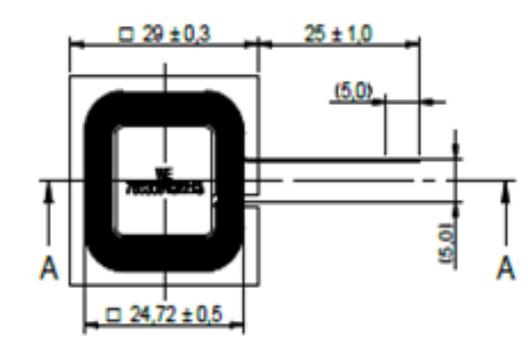

Figure 6. WE-WPCC wireless charging coil

\subsection{Subsystem design}

\subsubsection{Timer circuit code used in dsp}

Output Compare PWM mode has been used in the DSP for the generation of switching logic for the MOSFETs. OC2CON Special function Register (SFR) has been used to select the operation mode of the Output Compare Module. Initially, OC2CON bits are reset. Then steps are followed to configuring it to Continuous Pulse Mode. Pulse Width starts time is written on OC2R and stop time on OC2RS compare registers. Next, OCM bits of OC2CON register $(<2: 0\rangle)$ is set to 101 to configuring it to Continuous Pulse Mode. Next the timer is enabled by setting the TON bit of the T3CON register. Timer 3 is used here and the PR3 register is used to set the PWM period. PTCON register is used to set the PWM mode. Here it has been set to Free Running Mode and the Pre-Scalar value of 1:1 concerning external clock has been selected by setting the register value. Complementary Mode along with dead time has been configured using the PWMCON1 register. PDC1, PDC2, PDC3 SFRs are used to specify duty cycle values for the PWM module. The LS bit of these registers determines whether the PWM edge occurs in the beginning. The program body for assigning the SFR values is as given.

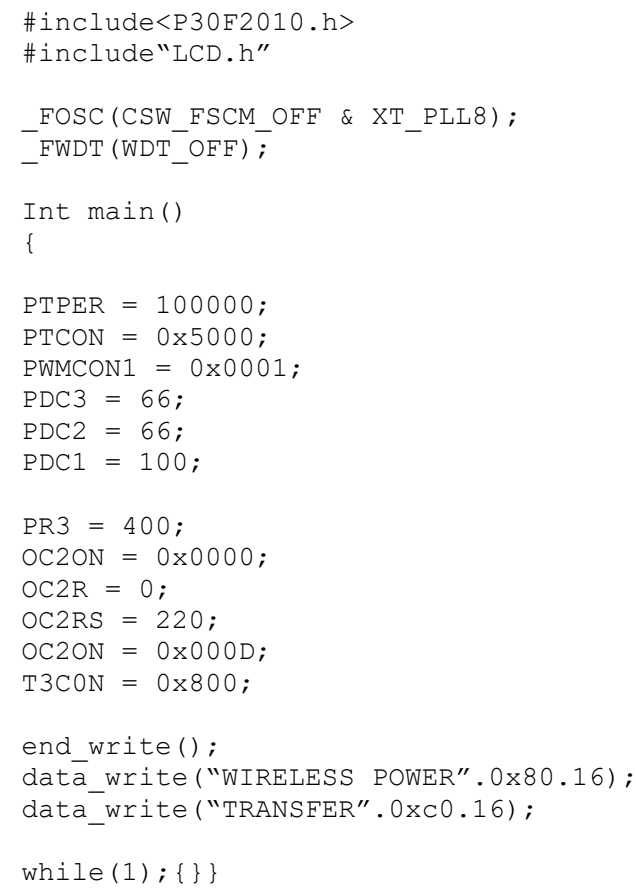




\subsubsection{Hardware assembly}

The circuit was initially assembled part wise on a breadboard and tested with the gradual increase in switching frequency. The input waveform to the transmitter coil was checked in oscilloscope and further design fine-tuning was done. The circuit was then assembled on a general-purpose printed circuit board (PCB). The PCB used has a clad of glass fiber with epoxy resin bond which has a higher frequency handling capacity. The PCB used is a two-layer, single-sided, with a separate power plane and signal plane. The finished circuit was soldered to the transmitter coil and power chords.

In the assembled system as an input source of power two transformers along with a bridge rectifier system has been used as shown in Figure 7(a). It is done so that direct supply from $220 \mathrm{~V}$ mains can be taken for the system but an adapter or a DC source of sufficing rating can also be used to power it up. One transformer provides for the main supply to the H-Bridge MOSFETs and other acts as a supplementary power source to supply the ICs. The receiver side consists of a bridge rectifier followed by a filter stage and ultimately fitted to supply for the charging of a Li-Ion battery. The output of the MOSFET is shown in Figure 7(b). The square waveform of $150 \mathrm{kHz}$ is produced. Output at the receiver end shown in Figure 8.

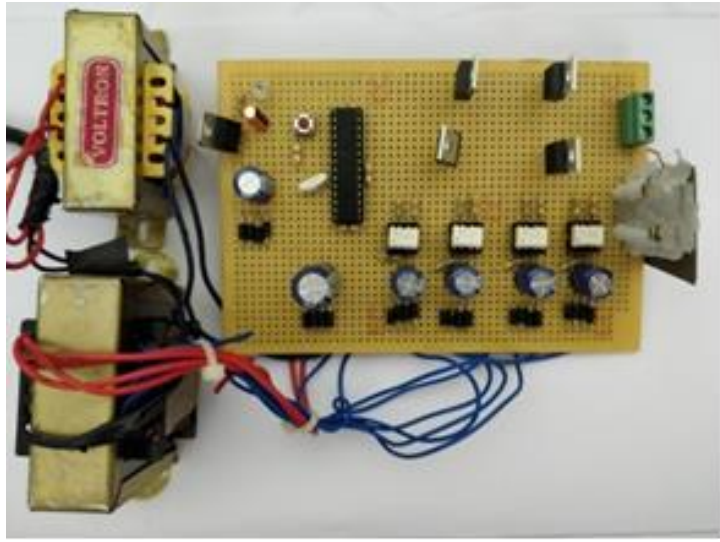

(a)

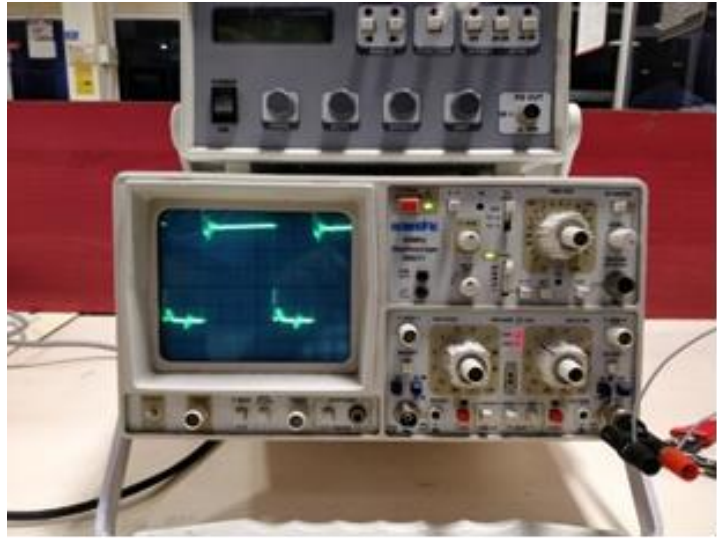

(b)

Figure 7. The Transmitter Side Configuration, (a) Hardware system, (b) Output

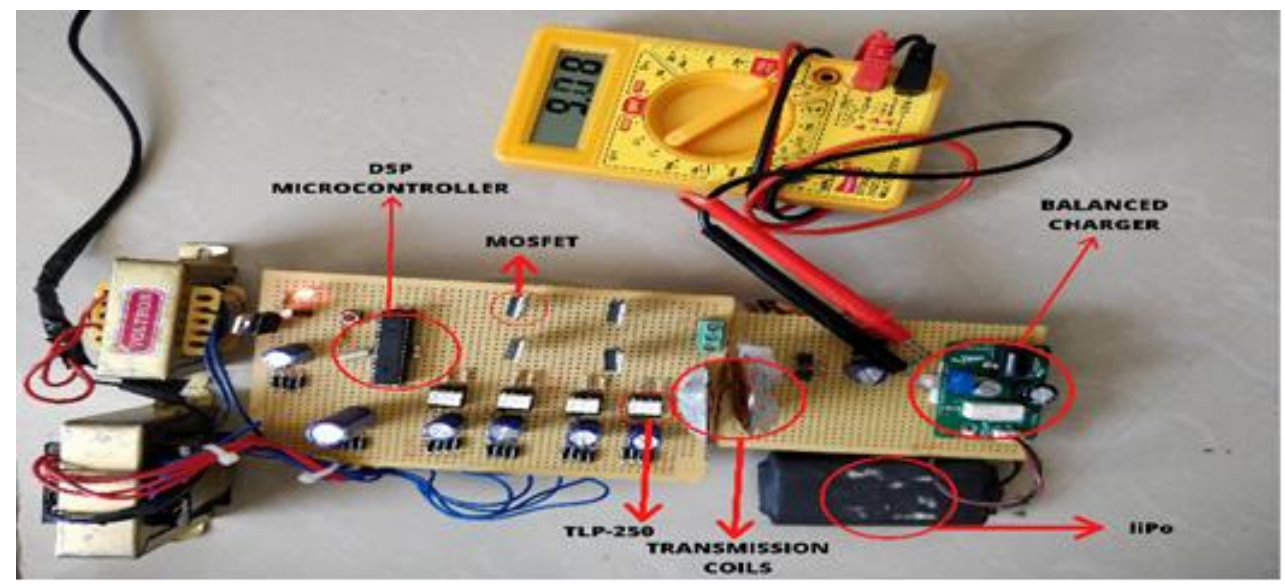

Figure 8. Output at the receiver end

\section{RESULTS}

We are receiving an output voltage of around 9 volts which is being used to charge the LiPo battery using the balanced charger module. This is however a prototype model that can be further developed into transmitting higher power by fine-tuning the inductive coupling and increasing the number of turns in the coil. This charging module can thus be implemented in charging stations for drones and various other robotic applications. 


\section{CONCLUSION}

The module we developed was tested with an input voltage of $15 \mathrm{~V}$ and has a handling capacity of $50 \mathrm{~V}$. The hardware developed is a prototype. Compared to existing models our system can transfer power successfully in the medium power range applications. The whole system hardware was assembled successfully and tests had been conducted related to the load handling capacity of the system. The scheme was proven to work and by the output values obtained. Visual observations made on the prototype assembled. The sensitivity of the scheme towards varying air gap length of the wireless coil was also tested

\section{REFERENCES}

[1] Mark Estabrook, "The convenience of wireless charging: It's just physics," White Paper, MediaTek, 2017.

[2] YevgenBarsugov, "Battery Cell Balancing: What to Balance and How," Texas Instruments, 2018.

[3] Mercier, Patrick P., and Anantha P. Chandrakasan, "Rapid Wireless Capacitor Charging Using a Multi-Tapped Inductively-Coupled Secondary Coil," IEEE Transactions on Circuits and Systems I: Regular Papers, vol. 60, no. 9, pp. 2263-2272, 2013.

[4] Xiao Lu, Ping Wang, Dusit Niyato, Dong In Kim, and Zhu Han, "Wireless Charging Technologies: Fundamentals, Standards and Network Applications," IEEE, 2015.

[5] Stephan Schaecher, Matthias Brandl, "Resonant Wireless Power Transfer," White Paper, Infineon Technologies, 2018,

[6] Nicholas Abbondante, "Wireless Power: Cutting the cord in today's mobile World," White Paper, Intertek.

[7] Dean Clark, "Considerations when Designing a Wireless Charging System," White-Paper, ABRACON.

[8] Freescale Semiconductor, "Coils used for Wireless Charging," Inc. Application Note, 2014.

[9] Joseph C. Stark III, M.E., "Wireless Power Transmission Utilizing a Phased Array of Tesla Coils," in Electrical Engineering Thesis, 2004.

[10] Lixin Shi, Zachary Kabelac, Dina Katabi, and David Perreault. s., "Wireless Power Hotspot that Charges All of your Devices," In Proceedings of the 21st Annual International Conference on Mobile Computing and Networking (MobiCom '15). ACM, New York, 2015.

[11] Chandrashekar DR, "High-Frequency Inverter Design Fundamentals," Magphy Systems, 2010.

[12] Jiang, Chaoqiang, "An Overview of Resonant Circuits for Wireless Power Transfer," Energies, vol. 10, pp. 894, 2017.

[13] Gui-Jia Su, "Converter Topologies for Wired and Wireless Chargers," Oak Ridge National Laboratory, 2011.

[14] Rui Jin, "Circuits and Systems for Efficient Portable-to-Portable Wireless Charging," MIT, 2014.

[15] Chen, Weitong, and Chunhua Liu, Christopher Lee, and Zhiqiang Shan, "Cost_Effectiveness Comparison of Coupler Designs of Wireless Power Transfer for Electric Vehicle Dynamic Charging,” Energies, vol. 9, no. 11,2016

[16] S Cui et al., "Study on efficiency of different topologies of magnetic coupled resonant wireless charging system," IOP Conf. Ser.: Earth Environ. Sci., vol. 93, 2017.

[17] TianqingLi, Xiangzhou Wang, Shuhua Zheng, and Chunhua Liu, "An Efficient Topology for Wireless Power Transfer Over A Wide Range of Loading Conditions," Energies MDPI, 2018.

[18] Paolo Batezzato, "Wireless Battery Charging," White-Paper, ST, Technology Tour, 2017.

[19] Stephan Schächer, MilkoPaolucci, "Wireless charging: advanced technology delivers consumer convenience," White Paper, Infineon Technologies AG, 2017.

[20] Robert Alexander Moffatt, "Wireless Transfer of Electric Power," MIT, 2009.

[21] M. Saravanan, A. Ramesh Babu, "High Power Density Multi-Mosfet-Based Series Resonant Inverter for Induction Heating Applications," International Journal of Power Electronics and Drive System (IJPEDS), vol. 7, no. 1, pp. 107-113, 2016.

[22] M. N. Tandjaoui, C. Benachaiba, O. abdelkhalek, B. Denai, Y. Mouloudi, "Characterisation and Modelling of Power Electronics Device," International Journal of Power Electronics and Drive System (IJPEDS), vol. 5, no. 2, pp. 135-14, 2014.

[23] Hussain Attia, Ali Sagafinia, "Novel Discrete Components Based Speed Controller for Induction Motor," International Journal of Power Electronics and Drive System (IJPEDS), vol. 7, no. 4, pp. 1075-1084, 2016.

[24] A. Ali, M.N.M Yasin, M.F.C. Husin, N.A.M Ahmad Hamb ali, "Design and analysis of 2-coil wireless power transfer (WPT) using magnetic coupling technique," International Journal of Power Electronics and Drive System (IJPEDS), vol. 10, no. 2, pp. 611-616, 2019.

[25] Masood Rehman, Perumal Nallagownden, Zuhairi Baharudin, "Efficiency investigation of SS and SP compensation topologies for wireless power transfer," International Journal of Power Electronics and Drive System (IJPEDS), vol. 10, no. 4, pp. 2157-2164, 2019. 


\section{BIOGRAPHIES OF AUTHORS}
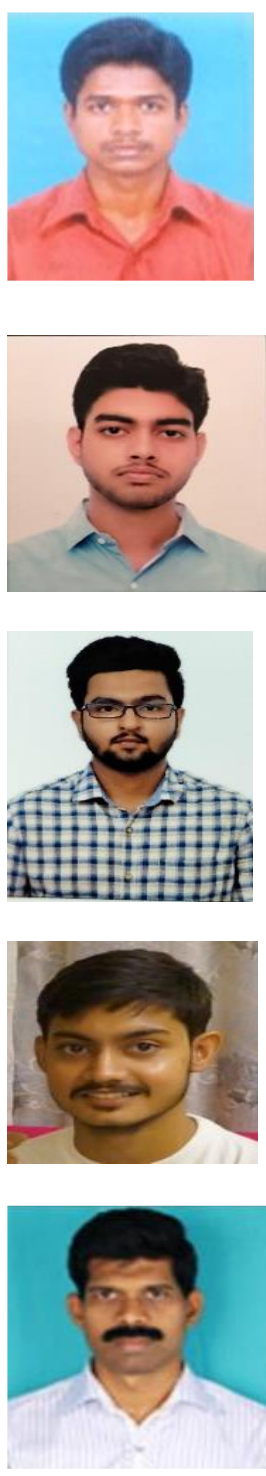

D. Karthikeyan was awarded a B.E. Degree from the Electrical and Electronic Engineering Department of A.I.H.T College, Chennai, India (affiliated to Anna University, Chennai, India), in 2009; and M.Tech. Graduated from SRMIST (formerly SRM University), Kattankulathur, India, in Power Electronics and Drives in 2013, he completed his Ph.D. in Multilevel Inverters in 2019. He currently works as an assistant professor in the SRMIST (formerly SRM University) Department of Electrical Engineering, Kattankulathur, and Chennai, India. His current research interests include power electronic multilevel inverters, AC drives, and DC drives. He is a member of various professional societies such as the IEEE, IET, and ISCA.

Sayon Koley, pursuing B.Tech from SRM Institute of Science and Technology with major in Electrical and Electronics Engineering. He has a subject interest in Power System Engineering. His current research interests include inverter designing and high-frequency topologies.

Mayukh Bagchi, pursuing B.Tech from SRM Institute of Science and Technology with major in Electrical and Electronics Engineering. He has a subject interest in experimental physics. His current research interests include wireless power transfer and particle accelerator design.

Avijit Bhattacharya, pursuing B.Tech from SRM Institute of Science and Technology with major in Electrical and Electronics Engineering. He has a subject interest in Electromagnetic Theory. His current research interests include High Voltage power supply designing and inverter designing.

Vijayakumar Krishnasamy received his B.E. and M.E. degrees from Annamalai University, Annamalai Nagar, India; and his Ph.D. degree from SRMIST (Formerly SRM University), Kattankulathur, India. He is presently working as a Professor and as the Head of the Department of Electrical and Electronics Engineering of SRMIST (Formerly SRM University). His current research interests include power system modeling, power electronics converters for grid-connected PV systems, computational intelligence applications in power systems, FACTS devices, and power quality. He has been awarded the Best Teacher Award in his department for the academic years 2004 and 2006. He is a member of various professional societies such as the IEEE, IET, FIE, ISTE, and ISCA. 